\title{
Design Optimization and Fabrication of Graphene/ J-aggregate Kretschmann-raether Devices for Refractive Index Sensing Using Plasmon-induced Transparency Phenomena
}

Amir Mohammad Rezaei Zanganeh

Islamic Azad University

Ali Farmani ( $\sim$ farmani.a@lu.ac.ir)

Lorestan University https://orcid.org/0000-0002-4842-0181

Mohammad Hazhir Mozaffari

Islamic Azad University

Ali Mir

Lorestan University

\section{Research Article}

Keywords: Fabrication of Graphene, Transparency Phenomena, magnetic field

Posted Date: October 21st, 2021

DOl: https://doi.org/10.21203/rs.3.rs-837103/v1

License: (c) (1) This work is licensed under a Creative Commons Attribution 4.0 International License.

Read Full License

Version of Record: A version of this preprint was published at Plasmonics on January 8th, 2022. See the published version at https://doi.org/10.1007/s11468-021-01591-7. 


\title{
Design optimization and fabrication of graphene/ J-aggregate Kretschmann-Raether devices for refractive index sensing using plasmon-induced transparency phenomena
}

\author{
Amir Mohammad Rezaei Zanganeh, ${ }^{1}$ Ali Farmani, ${ }^{2, \dagger}$ Mohammad Hazhir \\ Mozaffari, ${ }^{1, *}$ and Ali Mir ${ }^{2}$
}

\begin{abstract}
${ }^{1}$ Department of Electrical Engineering, Sanandaj Branch, Islamic Azad University, Sanandaj, Iran ${ }^{2}$ Optoelectronics research center, Department of Electrical Engineering, Lorestan University, Iran †Farmani.a@1u.ac.ir
\end{abstract}

Mh.mozaffari@iausdj.ac.ir

\begin{abstract}
Here, a novel plasmon-induced transparency (PIT) sensing platform based on a Kretschmann-Raether configuration with graphene/ J-aggregate materials is proposed. The $\mathrm{J}$-aggregate material, despite its dielectric optical properties, can strongly confine the surface wave-like metal layers. These features promise to highly enlarge the range of plasmonic sensing devices. Therefore, the sensing parameters have been numerically and experimentally investigated using the finite-difference time-domain (FDTD) method and atomic force microscopy (AFM). The results show that the PIT resonance of the structure has a sharp reflection, in turn, leads to high sensitivity. To deep benchmark the structure the effects of the structural parameters and environmental variables such as temperature and magnetic field on the sensing properties of the device are analyzed in detail. The maximum sensitivity is obtained as high as 1400 angle per refractive-index unit (RIU) with an extra high figure of merit of $36 \mathrm{RIU}^{-1}$ around the PIT resonance angle of $53^{\circ}$. By considering the magnetic field of $0.01 \mathrm{~T}$ and graphene chemical potential of $\mu_{\mathrm{c}}=0.4 \mathrm{eV}$, and environmental room temperature, the proposed structure may potentially be applied in advanced off-chip PIT sensors.
\end{abstract}

\section{Introduction}

Sensors, as an analytical and efficient tool, play a significant role in different fields, including medical science $^{1}$, military ${ }^{2}$, industrial ${ }^{3}$, and agriculture ${ }^{4}$, etc.Given the frequent applications of sensors, their environmental limitations, and diagnostic conditions of the sensor, different types of sensors have been developed so far. One of the most effective methods is to use light as an optical sensing probe. The history of the optical sensor applications based on the characteristics of absorption, emission, and fluorescence dates back to the 16th century ${ }^{5}$. Today, $\mathrm{THz}$ sensing has developed as a promising technique for biomedical research and material identification concerning terahertz fingerprints of DNA molecules, chemicals, and terahertz spectroscopy in the time and frequency domains ${ }^{6}$. The strong absorption lines in the wavelength range of 3-8 $\mu \mathrm{m}$ and the mid-infrared spectral range provide a significant opportunity for the gas detectors ${ }^{7}$. one of the fundamental characteristics of sensors is their size. Therefore, present sensors are mostly designed on a nanometer scale, and the competition is over the design of smaller sensors ${ }^{8-10}$. Accuracy of measurements, which is directly proportional to sensor sensitivity, is a factor that can play an important role in choosing a sensor. Sensitivity is defined as the ratio of spectral displacement to changes in the 
effective refractive index. This displacement can be associated with changing the center of the amplitude of the transmitted light wavelength or changing the time interval of the transmitted light wavelength relative to the ground state. The higher the sensor sensitivity, the more accurate it is ${ }^{10-12}$. Another factor in evaluating a sensor is its figure of merit (FOM). At a constant wavelength, FOM is defined as the ratio of the light transmission changes at a constant wavelength $(\Delta \mathrm{T})$ to the change in refractive index $(\Delta \mathrm{n})$, divided by the transmission at a certain wavelength $(T)^{10,12,13}$. Many studies have been conducted at certain temperatures, so one of our priorities is that the sensor will continue its diagnostic function with sufficient accuracy at room temperature ${ }^{14}$. Another key factor in evaluating a sensor is labeled or label-free. If the sensor detects a specific analyte, it is a labeled sensor, and if it detects any analyte, it is a label-free sensor ${ }^{15,16}$. Label-free, high-sensitivity, cost-effective, small-scale, real-time optical sensors with biomedical applications can detect multiple analytes quickly ${ }^{17}$.

Ignacio and coworkers have recently developed an optimized D-shaped optical fiber (125 $\mu \mathrm{m}$ in diameter) with a nano-coating ${ }^{18}$. They showed that by optimizing the width, thickness, and refractive index of the nano-coating, the losses in the resonant mode could be reduced at the full width at half maximum (FWHM). By increasing the real part of the refractive index, the resonance width can be reduced to $24.8 \mathrm{~nm}$, FOM can be increased up to $709.4 \mathrm{RIU}^{-1}$. In environments with a refractive index of 1.3 to 1.335 , the sensitivity is $1566 \mathrm{~nm} / \mathrm{RIU}$. Talebifard et al. have investigated the sensitivity of a Silicon-On-Insulator (SOI) strip waveguide sensor ${ }^{19}$. They achieved the highest peak sensitivity of $270 \mathrm{~nm} / \mathrm{RIU}$ by changing the waveguide thickness to $165 \mathrm{~nm}$ to obtain the highest sensitivity in the quasi-TM mode. Before this study, changes in the waveguide thickness to achieve maximum sensitivity were investigated for a slab waveguide but not for a waveguide amplifier that has different characteristics. Recently, Sheng et al. investigated the terahertz metamaterials using three-dimensional double split-ring resonators ${ }^{20}$. Metamaterials are artificial electromagnetic structures not found in nature and exhibit many unique electrical and magnetic properties, including synthetic magnetism ${ }^{21}$, thyroid response, negative refractive index ${ }^{22}$. Due to the high electromagnetic response of metamaterials in the frequency range of terahertz, their regulation and control in the sensing field are of great importance. They were able to regulate the reflection coefficients in the terahertz range by changing the physical structure of the ring resonator. Free spectrum range (FSR) can be tuned from $0.20 \mathrm{THz}$ to $0.75 \mathrm{THz}$. Recent research has always been associated with innovation and optimization in different fields of optical sensors, so researchers try to open new research windows to improve different parameters in the field of sensors. Accordingly, the plasmonic field has been introduced to reduce the dimensions, increase the sensitivity ${ }^{23}$, and also integration ${ }^{23-25}$. At its most fundamental, the field of plasmonics can be described as the study of plasmons, or collective charge oscillations, in conducting or even semiconducting materials ${ }^{26}$. The interaction between aggregate charge fluctuations and electromagnetic radiation on the surface of conductive material will lead to the emergence of a phenomenon most attractive in the plasmonic field. The plasmonic structure can be divided into two parts. The first part is known as the localized surface plasmon resonance (LSPR) that leads to the very strong interaction between light fields in a focused range ${ }^{27}$. Accordingly, localized plasmon-based sensors are suitable for detecting changes in the local refractive index caused by the adsorption of targets ${ }^{28}$. Recently, a hybrid structure has been used to enhance the FOM of plasmonic sensors ${ }^{27}$. Due to the extremely high sensitivity and the large linewidth in the structure of the localized surface plasmon and the increased bandwidth that leads to reduced FOM, Liu et al. could provide a plasmonic-photonic structure by applying a diagonal light. The plasmonic-photonic structure had the maximum spectral quality coefficient in a range of 92 to1375, leading to a FOM of $1337 \mathrm{RIU}^{-1}$. Recently, a practical approach has been proposed for designing optical sensors using an aluminum-based plasmonic nanoring array ${ }^{29}$. They introduced an effective refractive index sensor at visible wavelengths based on the surface plasmon resonance. Features of this research include cost-effectiveness, ease of construction, and compatibility with CMOS. Salemizadeh et al. has achieved, 
the sensor sensitivity and FOM were $408 \mathrm{~nm} / \mathrm{RIU}$ and $5.88 \mathrm{RIU}^{-1}$. The second part is known as the surface plasmon polaritons (SPPs). The interaction between free electrons oscillating on a metal surface and photons will polarize the SPPs ${ }^{30}$. SPPs are electromagnetic waves that propagate across the surface of an insulating-metal interface in a horizontal field excited by polar magnetic light perpendicular to the interface. The performance (e.g., footprint and light confinement) of the traditional optical systems are inevitably restrained by the diffraction limit of light. SPPs enable to confine light at sub-wavelength scale and overcome the diffraction limit of light, providing an excellent platform for nanoscale plasmonic control and elements $^{31,32}$. Due to the high sensitivity of SPPs to the surrounding environment, which is associated with the reduced linewidth, Bowen et. al. has designed a plasmonic sensor ( $\mu \mathrm{m}$ in size) with extremely high FOM due to resonant linewidth less than $3 \mathrm{~nm}$, by providing a structure consisting of a silicon substrate, a mid-layer of the photoresistor, a surface gold film with a thickness of $100 \mathrm{~nm}$, and periodic arrays of 620 $\mathrm{nm}^{33}$. The linewidth less than $8 \mathrm{~nm}$ was obtained in the wavelength range of 600-960 nm, and FOM reached $760 \mathrm{RIU}^{-1}$ at a wavelength of $960 \mathrm{~nm}$ due to a linewidth of $3 \mathrm{~nm}$. Plasmon-induced transparency (PIT) has recently been studied to increase the sensitivity and FOM ${ }^{12}$, control the transmission spectrum, and adjust the transparent window ${ }^{12,34,35}$. It is a classic analog of electromagnetically induced transparency (EIT), which occurs in atomic systems and creates a narrow transparent window in the main absorption spectrum ${ }^{31}$. In the transparent window, the scattering properties of the atomic environment can be significantly modified, which has promising applications in nonlinear optics, optical data storage, nanophotonics, and integrated photonic devices ${ }^{36}$. PIT has resolved the serious implementation constraints and has led researchers to take this approach ${ }^{37}$. In recent years, Matsunaga and coworkers have investigated the PIT in a metal (gold)-insulator (silicon)-metal (silver) (MIM)structure by Fano resonance between the MIM waveguide mode and the SPP resonance mode by creating a transparent window in an opaque area ${ }^{38}$. Fano resonance, as a fundamental resonant effect, creates a transparent window due to the interference of a localized state and a continuous state ${ }^{39}$. Combining Fano resonance with the plasmonic structures enables ultra-compact optical devices to be accomplished with fully integrated optics. Matsunaga group could control the transparent window by providing a MIM structure and using a prism at the top of the structure in the Kretschmann configuration and varying the thickness of the gold and silver layers. Recently, Zhang et al. introduced a Fano resonance-based refractive index sensor in a plasmonic waveguide surrounded by two resonance rings ${ }^{24}$. They could control the transmission spectrum and the transparent window by changing the dimensions of the defect, the radius of the resonance rings, the waveguide width, and the refractive index of the medium. In this regard, the sensitivity, sensor transparency, and FOM were determined theoretically as $1160 \mathrm{~nm} / \mathrm{RIU}, 8.62 \times 10^{-5} \mathrm{RIU}$, and $73 \mathrm{RIU}^{-1}$, respectively. The transmission spectrum was evaluated in a wavelength range of 0.8 to $1.3 \mu \mathrm{m}$. Jing Chen et al. theoretically demonstrated good efficiency for a refractive index sensor consisting of a two-dimensional array of gold nanoparticles mounted on a narrow dielectric waveguide ${ }^{40}$. A quasi-sharp Fano resonance due to the strong interference between the LSPR in excited gold nanoparticles and the light-emitting waveguide state in the dielectric plate gives a sensitivity of $250 \mathrm{~nm} / \mathrm{RIU}$ and a FOM of $28 \mathrm{RIU}^{-1}$. The transmission spectra were also examined by changing the dielectric media. Another method that has been studied to improve the sensitivity of SPR sensors in the transparent window is Goos-Hänchen $(\mathrm{GH})$ shifts $^{41}$. It is evident that when an electromagnetic wave is reflected completely from the interface between two different media, a small lateral displacement occurs between the electromagnetic wave of practical reflection and the electromagnetic wave of geometry reflection ${ }^{42}$. Positive and negative GH shifts in light reflection and transmission correspond to the positive or negative refractive index of the medium, respectively. Accordingly, using EIT, without medium disturbance and with nearly negligible light beam absorption in the medium, positive and negative GH shifts can be accomplished in light reflection and transmission ${ }^{43}$. Thus, Chery et al. introduced a GH angular shift sensor using a gold film excited by SPR in the Kretschmann configuration ${ }^{44}$. The glass slide with the $50 \mathrm{~nm} \mathrm{Au}$ film thickness was attached to the hemispherical prism using an index-matching oil (n 
$=1.518)$. Chery et al. focused the incident beam in the middle of the narrow beam to increase the GH angular shift. They experimentally and computationally investigated the reflection properties of $\mathrm{GH}$ angular shift and showed a very sharp resonance at a resonance angle of $43.8^{\circ}$ in the measurement of SPR. Excessive expansion of the wave vector under a focusing geometry reduces the effective excitation of surface plasmons and increases the GH angular shift. Since the research done to control the transparent window has generally been done by modifying the structure physically ${ }^{12,34,38,45}$ or changing the refractive inde ${ }^{36}$, so no changes can be made in the transparent window after constructing the sensor. Studies have shown that the metal choice and its thickness are very important for SPR. Accordingly, the major metals used for surface plasmon resonance are silver, gold ${ }^{33,38,44,46}$, and aluminum ${ }^{29}$. By changing their size and shape, plasmonic control and behavior of these materials are accomplished. Such structures have high ohmic and non-adjustable losses, especially in the visible light wavelength ${ }^{47}$. Besides, there are limitations to the transmission length of surface plasmons between metal and dielectric. For this purpose, graphene has been introduced as an important candidate in the field of plasmonics, which is known as a two-dimensional material derived from carbon atoms, with a honeycomb structure and properties such as the adjustability to control the energy gap by changing the chemical potential or excitation voltage ${ }^{48}$, the single-atom thickness ${ }^{49}$, higher velocity due to scattering and lower number of collisions. More importantly, in the frequency range of terahertz photons are easily coupled with graphene surface plasmon waves (which is associated with significant properties $)^{50}$. Yuwen and coworkers could control PIT by varying the energy gap in a range of $0.25-0.85 \mathrm{eV}$ in a frequency range of 15 to $26 \mathrm{THz}$ without disturbing the existing physical structure and symmetry using graphene ribbons on a silicon substrate ${ }^{51}$. In addition to controlling the transparent window, they evaluated the linewidth in the range of Fermi level shift. The width of this structure was $1360 \mathrm{~nm}$, and the width of graphene ribbons was $580 \mathrm{~nm}$. Qichang et al. studied the effect of PIT numerically and theoretically on the metamorphic structure of graphene consisting of a resonator, graphene cut-wire, and two separate ring resonators in the mid-infrared region ${ }^{52}$. Compared to metal-based metamaterials, the effect of PIT realized in this study through the proposed metamorphic can provide more flexibility without the addition of active ingredients and structural changes, using the adjustability of the complex surface conductivity of graphene. In this study, the transmission spectrum in a range of 6.08-8.17 $\mu \mathrm{m}$ and the appearance of a transparent window were also investigated, in addition to changes in the energy gap due to changes in the physical dimensions of graphene and changes in refractive index.

To enhance the sensitivity, recently, J-aggregates nanomaterials are proposed which is suitable for designing off-chip sensors. Although J-aggregates nanomaterials have dielectric properties, in some wavelengths their dielectric constants are negative and therefore, can be used for the excitation of plasmon waves working at optical frequencies. One of the best candidates for plasmonics nanosensors is white graphene. Its optical properties can be changed in Retarded-regime and tend to negative values which introduce white graphene as a fantastic J-aggregates nanomaterial.

In this work, to improve the performance of the PIT Kretschmann-Raether-based sensors, for the first time to the best of our knowledge, a graphene/white graphene structure has been applied to construct the PIT refractive index sensor. Compared to a structure based on an on-chip, considering the application of Jaggregates, the strong light-matter interaction and therefore, high sensitivity can be obtained. The designed structure numerically and experimentally investigated. The results show that the proposed device can find potential applications in off-chip sensors. 


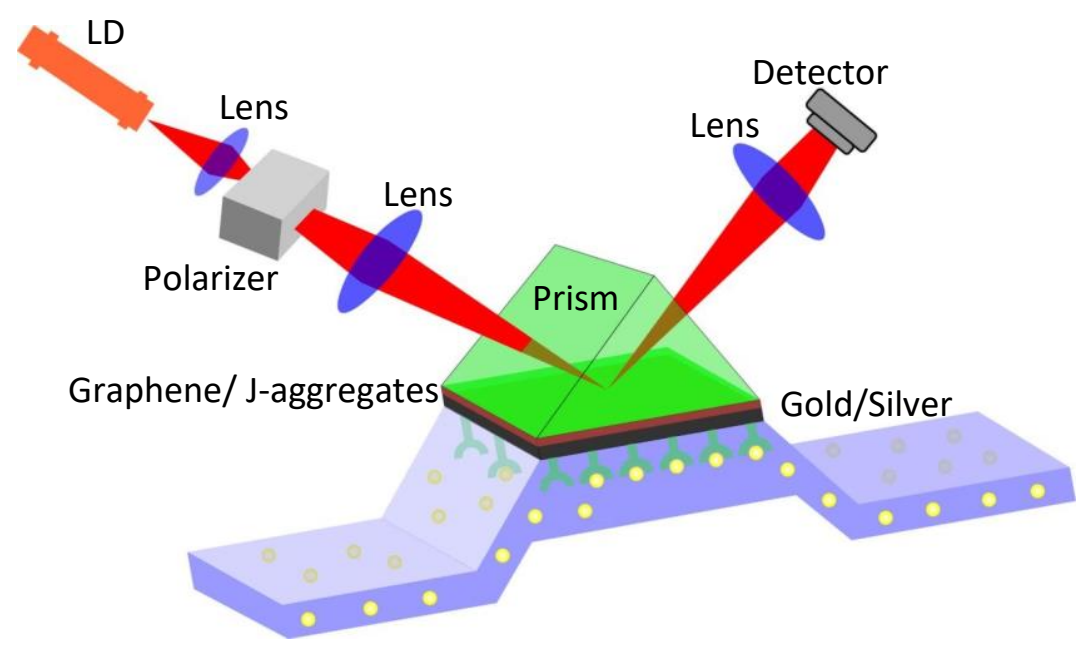

Figure 1. 3D-structure of the Kretschmann-Raether refractive index sensor

\section{Results}

Fig.1 illustrated the 3D-view of the proposed refractive index sensor using graphene plasmon-induced transparency phenomena. As shown, the proposed Kretschmann-Raether structure is formed by a prism layer for enhancing light-matter interaction, in turn, leads to the excitation of electromagnetic waves. Graphene layers are used with tunable optical properties for sensing of different refractive index. Also, considering the aim of this work, environmental effects via graphene can be monitored and the results will be obtained. White graphene as a J-aggregate layer can highly enhance the light-matter interaction. Besides, silver/gold metals for interaction between surface waves and incident power are applied.

The Kretschmann-Raether structure is assumed to be illuminated by an incident light with visible to NIR wavelengths, and the incident angle of $\square$ is controlled by a step motor to provide the resonance reign for PIT excitation. By applying graphene/white graphene as a refractive index sensing media, both of TE (TM) modes in the Kretschmann-Raether structure can be harnessed to detect different refractive indices. As the refractive index of the environment is changed, therefore, the reflection of PIT value and also resonance wavelength is chirped. With the above behavior, the different refractive indices can be easily detected with an extra-high sensitivity. This is the principle operation of the PIT refractive index sensor.

Next, the optical and geometrical properties of the Kretschmann-Raether structure are provided in Table.1. The application of these noble metals can be a help to increases both parameters including sensitivity and stability of the sensor. Because of the difference in the propagation constant of the environmental materials and graphene surface wave a dielectric prism is employed. Strong light-matter interaction is met by considering the J-aggregates behavior of white graphene. Also, this situation can provide a highly sensitive structure whose properties can be harnessed by tunable optical properties of graphene. 


\begin{tabular}{|c|c|c|}
\hline Layers & area $\left(\mu \mathrm{m}^{2}\right)$ & Thickness $(\mu \mathrm{m})$ \\
\hline Prism & $60 \times 60$ & 200 \\
\hline Graphene & $60 \times 60$ & $0.35 \times 10^{-3}$ \\
\hline White graphene & $60 \times 60$ & $1 \times 10^{-3}$ \\
\hline Gold/Silver & $60 \times 60$ & 0.1 \\
\hline
\end{tabular}

Table 1. Structural parameters of the Kretschmann-Raether structure

Here, the graphene layer is applied as an anisotropic medium, defined by a tensor formula

$$
\begin{aligned}
\sigma^{\leftrightarrow}\left(\omega, \mu_{c}\left(\mathbf{E}_{\mathbf{0}}\right), \Gamma, \mathrm{T}, \mathbf{B}_{\mathbf{0}}\right) & = \\
\hat{\mathbf{x}} \hat{\mathbf{x}} \sigma_{x x}+\hat{\mathbf{x}} \hat{\mathbf{y}} \sigma_{x y}+\hat{\mathbf{y}} \hat{\mathbf{x}} \sigma_{y x}+\hat{\mathbf{y}} \hat{\mathbf{y}} \sigma_{y y} & =\left(\begin{array}{cc}
\sigma_{x x} & \sigma_{x y} \\
\sigma_{y x} & \sigma_{y y}
\end{array}\right)
\end{aligned}
$$

where $\omega$ refers to the angular frequency, $\mu_{c}$ refers the chemical potential, $\mathbf{E}_{\mathbf{0}}$ is applied voltage, $\Gamma$ refers to the scattering rate $(\Gamma=1 / 2 \tau)$ ( $\tau$ is the relaxation time), $\mathrm{T}$ refers to temperature and $\mathbf{B}_{\mathbf{0}}$ refers to the magnetic field. Here, $\sigma_{x x}\left(\sigma_{y y}\right)$ and $\sigma_{x y}\left(\sigma_{y x}\right)$ are longitudinal (diagonal) and transverse (off-diagonal) conductivities, respectively and calculated by Kubo relations:

$$
\begin{gathered}
\sigma_{x x}\left(\mu_{c}\left(E_{0}\right), B_{0}\right)=\frac{e^{2} v_{f}^{2}\left|e B_{0}\right|(\omega-j 2 \Gamma) \hbar}{-j \pi} \times \sum_{n=0}^{\infty} \\
\left\{\frac{f_{d}\left(M_{n}\right)-f_{d}\left(M_{n+1}\right)+f_{d}\left(-M_{n+1}\right)-f_{d}\left(-M_{n}\right)}{\left(M_{n+1}-M_{n}\right)^{2}-(\omega-j 2 \Gamma)^{2} \hbar^{2}} \times\left(1-\frac{\Delta^{2}}{M_{n} M_{n+1}}\right)\right. \\
\times \frac{1}{M_{n+1}-M_{n}}+\frac{f_{d}\left(-M_{n}\right)-f_{d}\left(M_{n+1}\right)+f_{d}\left(-M_{n+1}\right)-f_{d}\left(M_{n}\right)}{\left(M_{n+1}-M_{n}\right)^{2}-(\omega-j 2 \Gamma)^{2} \hbar^{2}} \\
\left.\times\left(1+\frac{\Delta^{2}}{M_{n} M_{n+1}}\right) \frac{1}{M_{n+1}+M_{n}}\right\} \\
\sigma_{x y}\left(\mu_{c}\left(E_{0}\right), B_{0}\right)=-\frac{e^{2} v_{f}^{2} e B_{0}}{\pi} \times \sum_{n=0}^{\infty}\left\{f_{d}\left(M_{n}\right)-f_{d}\left(M_{n+1}\right)-\right. \\
\left.f_{d}\left(-M_{n+1}\right)+f_{d}\left(-M_{n}\right)\right\} \times\left\{\left(1-\frac{\Delta^{2}}{M_{n} M_{n+1}}\right) \times\right. \\
\frac{1}{\left(M_{n+1}-M_{n}\right)^{2}-(\omega-j 2 \Gamma)^{2} \hbar^{2}}+\left(1+\frac{\Delta^{2}}{M_{n} M_{n+1}}\right) \times \\
\left.\frac{1}{\left(M_{n+1}+M_{n}\right)^{2}-(\omega-j 2 \Gamma)^{2} \hbar^{2}}\right\}
\end{gathered}
$$

here $M_{n}$ refers to the quantized Landau Levels by quantum numbers $n(n=0,1,2, \ldots)$ and it is calculated by:

$M_{n}=\sqrt{\Delta^{2}+2 n v_{f}^{2}\left|e B_{0}\right| \hbar}$

And $f_{d}(M)$ refers to the Fermi-Dirac distribution:

$f_{d}\left(M_{n}\right)=\frac{1}{1+e^{\left(M_{n}-\mu_{c}\right) / k_{B} T}}$ 
$\hbar$ refers to the reduced Plank constant, $-e$ refers to the electron charge, $v_{f}$ refers to the Fermi velocity $\left(\approx 10^{6} \mathrm{~m} / \mathrm{s}\right)$ and $\Delta$ refers to the excitonic energy gap.

Here, two conditions are considered. As the magnetic field is ignored and only the voltage $\left(\mu_{\mathrm{c}} \neq 0\right)$ are considered. The optical conductivity of graphene in this case is:

$$
\begin{gathered}
\sigma_{L}\left(\mu_{c}\left(E_{0}\right)\right)=\frac{j e^{2}(\omega-j 2 \Gamma)}{\pi \hbar^{2}}\left[\frac{1}{(\omega-j 2 \Gamma)^{2}} \times\right. \\
\int_{0}^{\infty} \varepsilon\left(\frac{\partial f_{d}(\varepsilon)}{\partial \varepsilon}-\frac{\partial f_{d}(-\varepsilon)}{\partial \varepsilon}\right) d \varepsilon- \\
\left.\int_{0}^{\infty} \frac{f_{d}(-\varepsilon)-f_{d}(\varepsilon)}{(\omega-j 2 \Gamma)^{2}-4\left(\frac{\varepsilon}{\hbar}\right)^{2}} d \varepsilon\right]=\sigma_{L, \text { intra }}+\sigma_{L, \text { inter }}
\end{gathered}
$$

where $f_{d}(\varepsilon)$ refers to the Fermi-Dirac distribution:

$f_{d}(\varepsilon)=\left(e^{\left(\varepsilon-\left|\mu_{c}\right|\right) / k_{B} T}+1\right)^{-1}$

Here, optical sensitivity can be categories in two terms of intraband and interband as expressed:

$\sigma_{L, \text { intra }}=-j \frac{e^{2} k_{B} T}{\pi \hbar^{2}(\omega-j 2 \Gamma)}\left(\frac{\mu_{c}}{k_{B} T}+2 \ln \left(e^{-\frac{\mu_{C}}{k_{B} T}}+1\right)\right)$

For $k_{B} T \ll\left|\mu_{c}\right|, \hbar \omega$ the interband conductivity can be calculated as:

$\sigma_{L, i n t e r} \simeq \frac{-j e^{2}}{4 \pi \hbar} \ln \left(\frac{2\left|\mu_{c}\right|-(\omega-j 2 \Gamma) \hbar}{2\left|\mu_{c}\right|+(\omega-j 2 \Gamma) \hbar}\right)$

To considering magnetic effects on sensing parameters, also, optical conductivities $\sigma_{L}$ and $\sigma_{H}$, are calculated:

$\sigma_{L}(\omega)=\sigma_{0} \frac{1+j \omega \tau}{(1+j \omega \tau)^{2}+\left(\tau \omega_{c}\right)^{2}}$

$\sigma_{H}(\omega)=\sigma_{0} \frac{\tau \omega_{c}}{(1+j \omega \tau)^{2}+\left(\tau \omega_{c}\right)^{2}}$

here $\tau=1 / 2 \Gamma$ refers to the relaxation time, $\sigma_{0}=\left(\frac{2 e^{2} \tau}{\pi \hbar^{2}}\right) k_{B} T \ln \left(2 \cosh \left(\frac{\mu_{c}}{2 k_{B} T}\right)\right)$ and $\omega_{c}=e B_{0} v_{f}^{2} / \mu_{c}$.

The real and imaginary parts of the graphene optical surface conductivity are plotted in Fig. 2 . As can be seen, by controlling external voltage on the graphene layer, the excitation of surface wave can be adjusted 

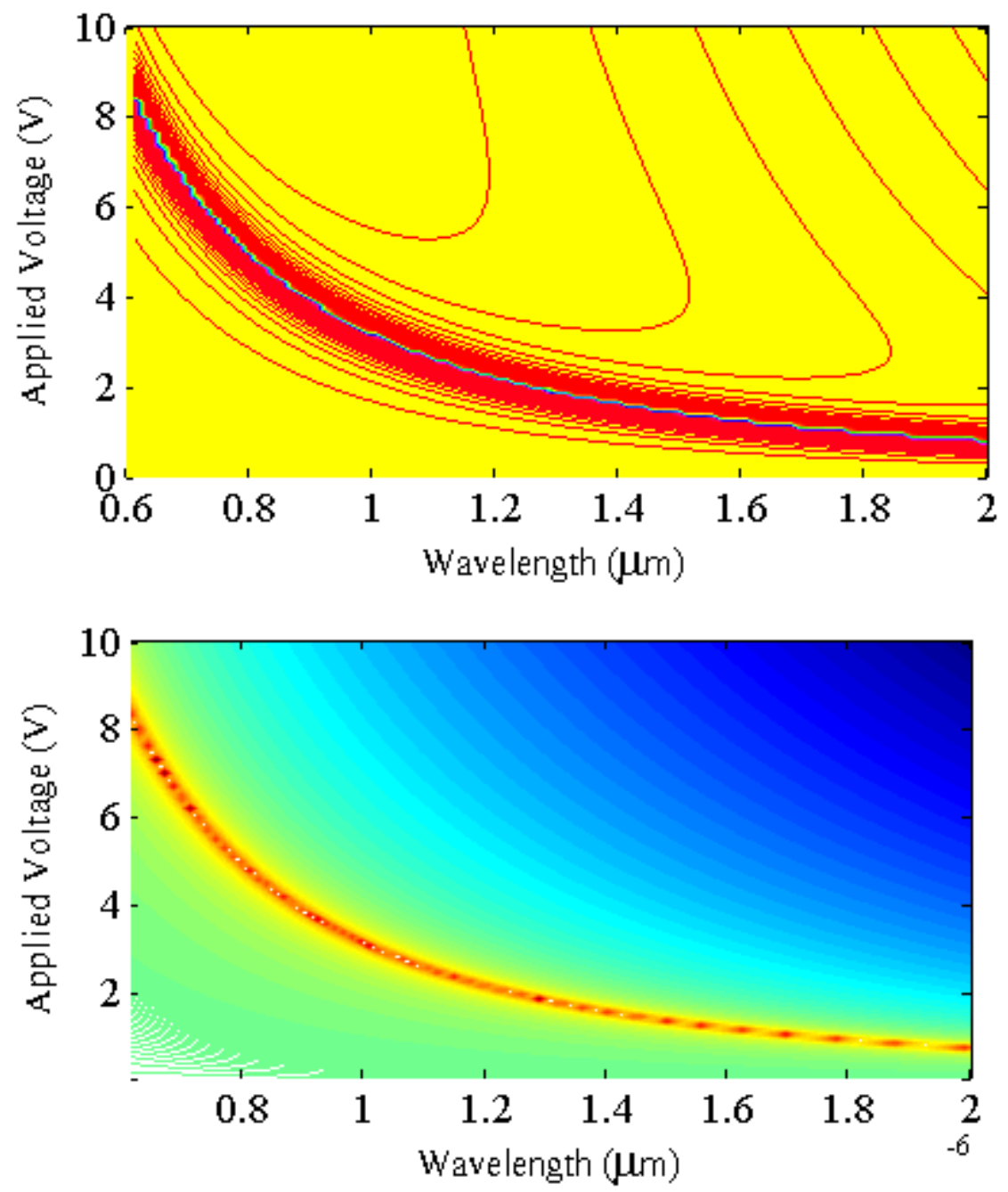

Figure 2. Real and imaginary parts of optical surface conductivity as a function of external voltage.

To analysis of the structure, the FDTD simulation method is exploited. To enhance the light-matter interaction it could be possible to obtain with J-aggregates LSRs within an arbitrary part of visible and NIR wavelengths. Also, J-aggregates propagate surface wave with long propagation length thus their LSRs is applied for the realization of high-performance sensors. The curves in Fig. 3 show the phase electric field. By considering PIT behavior, the $\left|\mathrm{E} / \mathrm{E}_{0}\right|^{2}$ should be higher than one. Here, the geometrical parameters are set as $\mathrm{L}=60 \mu \mathrm{m}, \mathrm{w}=60 \mu \mathrm{m}$, and $\mathrm{n}_{\mathrm{a}}=1$ so that the PIT resonance angle could be achieved near $53^{\circ} \mathrm{C}$. As can be seen, PIT with sharp resonance appears in the magnitude spectrum with value of 8 . Therefore, by considering this essential condition different cases are investigated. 

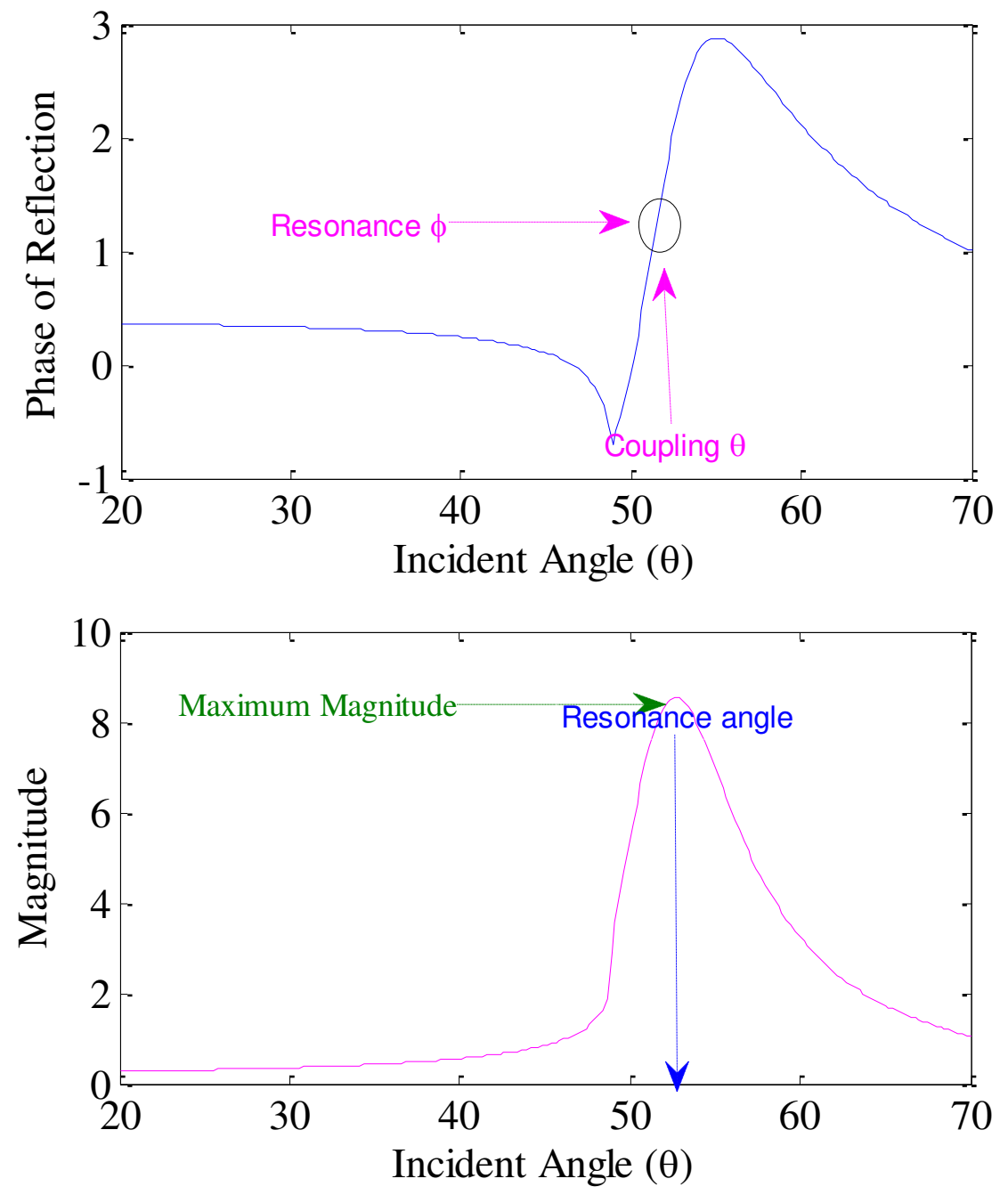

Figure 3. The calculated phase of reflection and magnitude for PIT condition.

\section{discussions}

\section{Chemical potential of graphene}

We investigated the effects of chemical potential on the electric field properties of the devices to find an optimum set of external parameters for the refractive index sensor. The excitation of surface wave is an important parameter of the devices, which can be defined by the FDTD method. To show the sensing behavior for two conditions of $\mu_{c}=0.45 \mathrm{eV}$ and $0.4 \mathrm{eV}$, the electric profiles of the field distributions of the structure are calculated in Fig. 4. As can be seen in Fig. 4(a) $\left(\mu_{\mathrm{c}}=0.4 \mathrm{eV}\right)$, most of the incident light is coupled to the boundary of graphene/white graphene, and the PIT phenomenon at the resonance wavelengths is achieved. However, in Fig. 4(b), (i.e, $\mu_{\mathrm{c}}=0.45 \mathrm{eV}$ ) almost no energy is coupled to the plasmonics layers. 

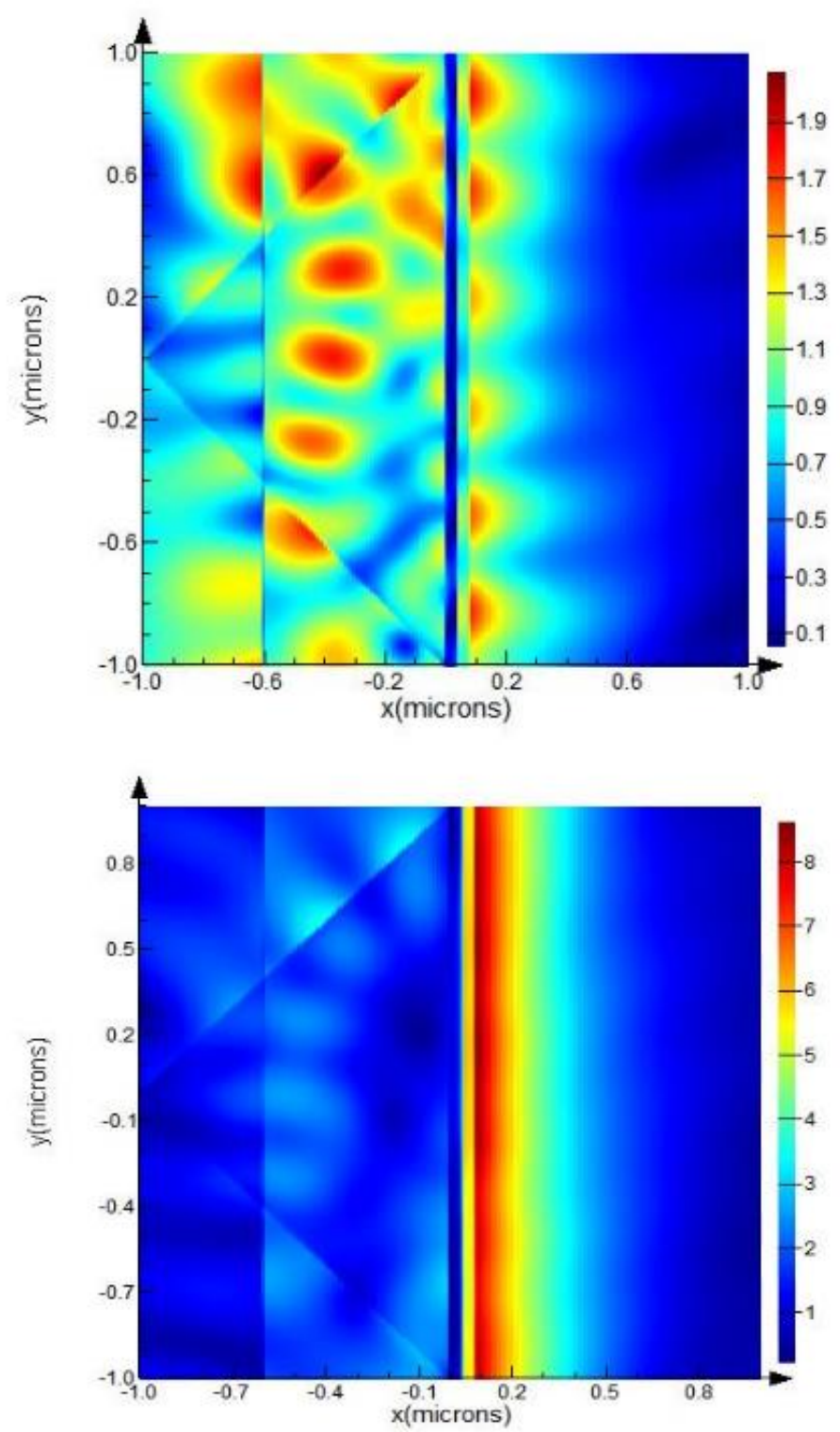

Figure 4. PIT condition considering $\square_{\mathrm{c}}=0.4 \mathrm{eV}$ and $\square_{\mathrm{c}}=0.45 \mathrm{eV}$.

\section{Near filed enhancements}

To analyze the effect of incident angle and chemical potential on the near-field enhancements properties $\left|\mathrm{E} / \mathrm{E}_{0}\right|^{2}$, the chemical potential is considered to be $0.4 \mathrm{eV}$ and $0.45 \mathrm{eV}$ and the incident angle is changed from 40 to 60 in steps of 1, whereas the other structural parameters were fixed. Fig. 5 illustrates the normalized near-field enhancements for two cases of chemical potential. As shown in Fig. 5, the PIT resonance of the two dark-bright modes obtains as chemical potential is $\mu \mathrm{c}=0.4 \mathrm{eV}$. Increasing the chemical potential results in weaker coupling and higher loss in the propagated light. Therefore, according to Fig. (3) and (4), a change in the chemical potential affects the PIT resonance. 

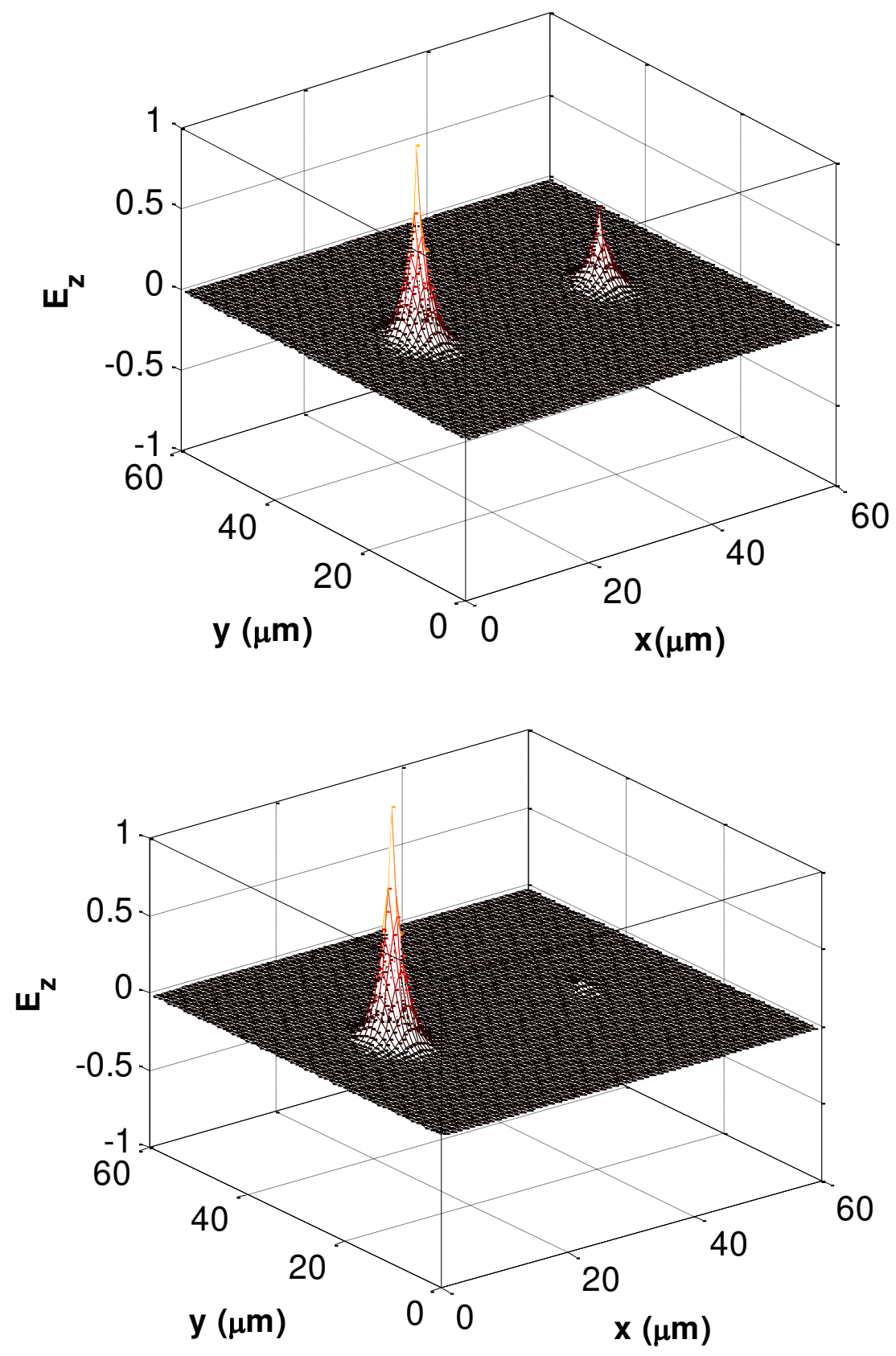

Figure 5. Near-field enhancements/ $\left.\mathrm{E}_{0}\right|^{2}$ as chemical potential is (a) $\mu \mathrm{c}=0.4 \mathrm{eV}$, (b) $\mu \mathrm{c}=0.45 \mathrm{eV}$

\section{Temperature effects}

To further study the performance of the refractive index sensor, we have investigated the effect of the external temperature (T) on the reflection spectrum. e $T$ is tuned from $27^{\circ} \mathrm{C}$ to $47^{\circ} \mathrm{C}$ in steps of 10 whereas other structural parameters are fixed. Fig. 6 illustrates the reflection spectrum of the Kretschmann-Raether as the incident angle is changed from 40 to 60 . As can be seen, by increasing the temperature, light 
confinement is decreased in the range of 40 to 50 , whereas their resonance points are relatively increasing. In conclusion, the variation of temperature is exactly detected.

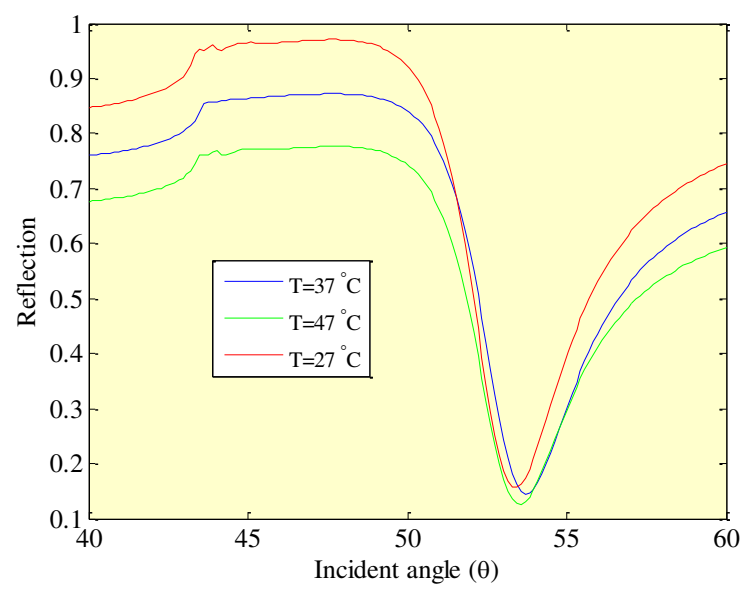

Figure 6. Calculation of reflection spectrum as temperature changes from $27^{\circ} \mathrm{C}$ to $47^{\circ} \mathrm{C}$.

\section{Refractive index sensing}

In order to study the performance of the proposed structure, here, we investigate how the environmental refractive index affects the sensing properties. To show the effects of the environmental changes on the sensing properties of the sensor, we tuned the refractive index from 1.0 to 1.1 in steps of 0.05 as chemical potential is $\mu \mathrm{c}=0.4 \mathrm{eV}$ for change of incident angle of 40 to 60 . Fig 7 shows the relationship between the resonance angle and the refractive index.

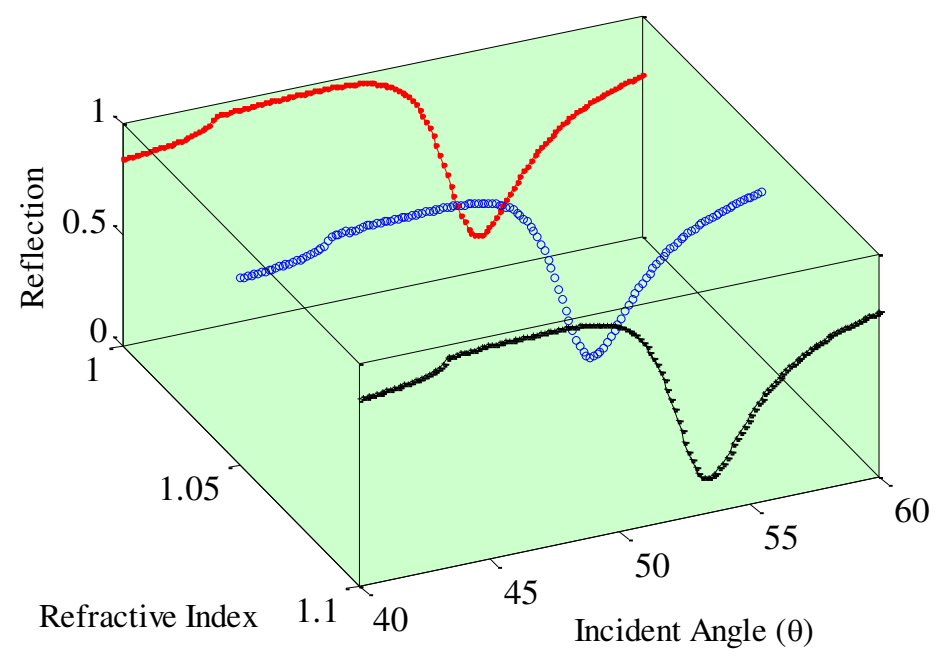

Figure 7. Reflection spectra of the sensor with different refractive indices.

As can be seen, by increasing the refractive index, the PIT resonance angle is blue shifted. This is the main mechanism of the proposed sensor for the detection of different refractive index with their small changes. 


\section{Magnetic field effects}

To discuss how the magnetic field affects the sensing properties, we applied different B of 0.01T, 0.02T, 0.03T, and 0.04T for different incident angles, while keeping other structural parameters fixed. Fig. 8 shows the resonance angle versus the incident angle for different external magnetic fields. As illustrated, the resonance angle has extra small changes for the different external magnetic fields. Therefore, the calculation under magnetic field has strong stability.

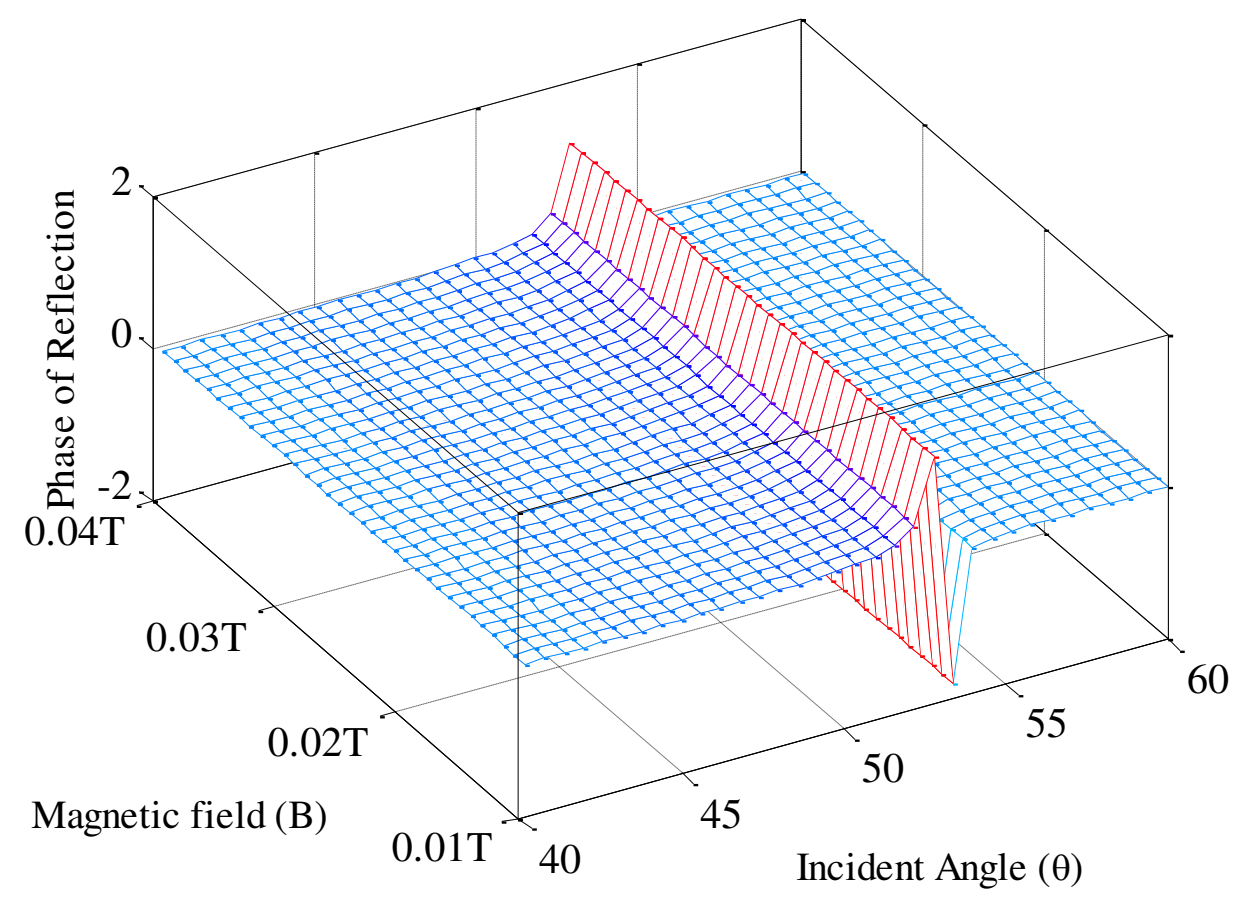

Figure 8. The phase of reflection of the sensor for different magnetic fields.

\section{Electric field effects}

To more exact discuss how the electric field affects the sensing properties, we applied $\mathrm{B}=0.01 \mathrm{~T}$ and control the chemical potential from $0.4 \mathrm{eV}$ to $0.47 \mathrm{eV}$. Here very important results are obtained. As can be seen in Fig. 9, as chemical potential is higher than $0.44 \mathrm{eV}$ no PIT mode is seen. Therefore, only dark mode is excited. In contrast, as chemical potential is lower than $0.44 \mathrm{eV}$ (Fig 9 (e) and (f)), PIT mode resonances. Moreover, as chemical potential is higher than $0.46 \mathrm{eV}$, the loss of surface plasmons increases. Therefore, for biasing the proposed structure, $\mu \mathrm{c}=0.4 \mathrm{eV}$ is the appropriate case. 

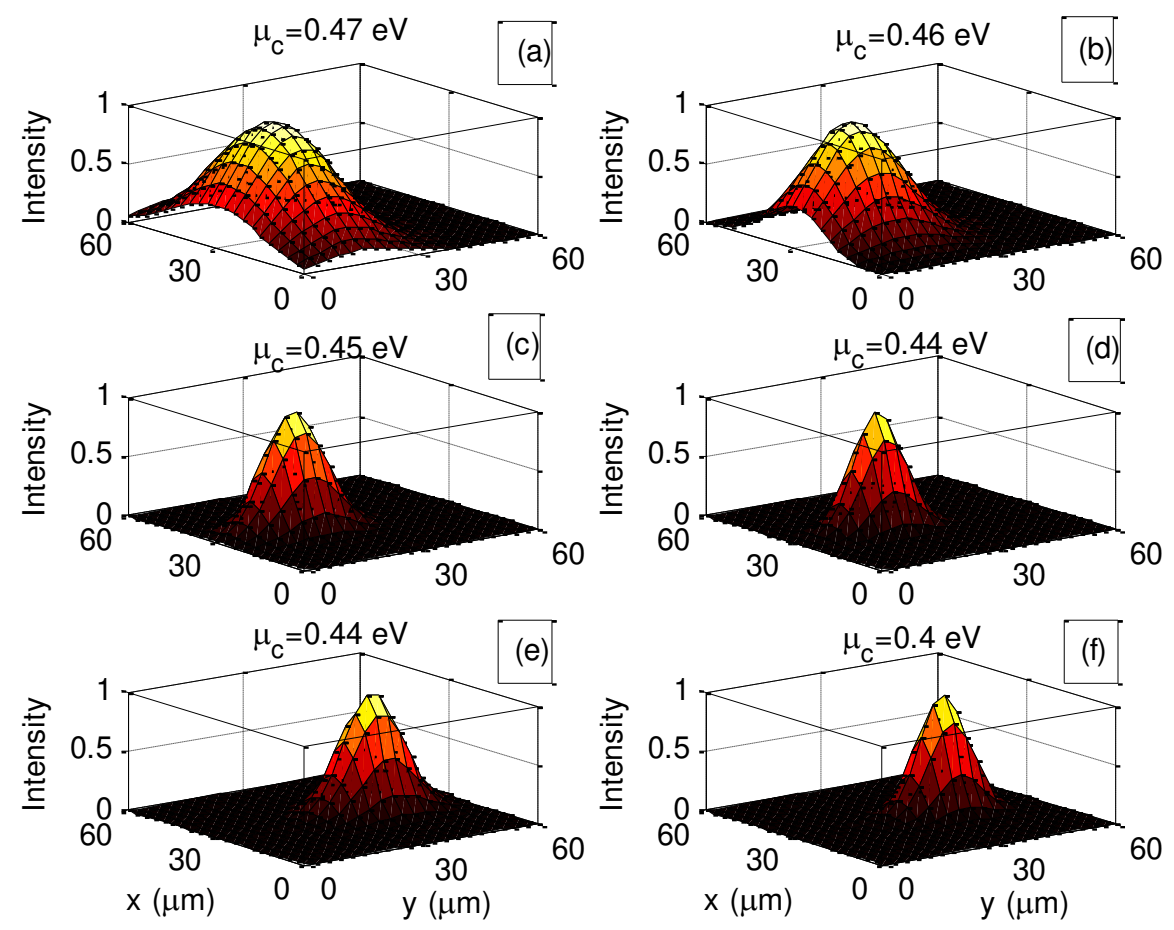

Figure 9. Normalized near-field enhancements/E0|2 for different chemical potential.

Therefore, refractive index sensing can be determined by measuring the shift of a certain resonance angle based on its linear relationship with chemical potential. Here, we study the performance parameters of the proposed structure considering the various parameters including sensitivity $(S)$ and figure of merit $(F O M)$. The sensitivity is calculated by:

$S=\Delta \theta / \Delta n$

here $\Delta \theta$ and $\Delta n$ are the spectrum shift and amount of the effective refractive index changes, respectively.

One of the crucial parameters in the design of refractive sensors is FOM which is presented to benchmark the operation of the system. Considering that one wavelength sources, like lasers, are widely applied in practice, the FOM factor is calculated by:

$F O M=\left[\left(\frac{\Delta \theta}{\Delta n}\right) / F W H M\right]=\frac{S S}{F W H M}$

here, FWHM refers to the full width at half maximum for surface resonance.

Here, sensitivity as a function of the graphene layer is examined. As can be seen, maximum sensitivity of 1400 A/RIU is obtained for four-layer graphene. By considering maximum sensitivity, FoM of 36 is achievable. 


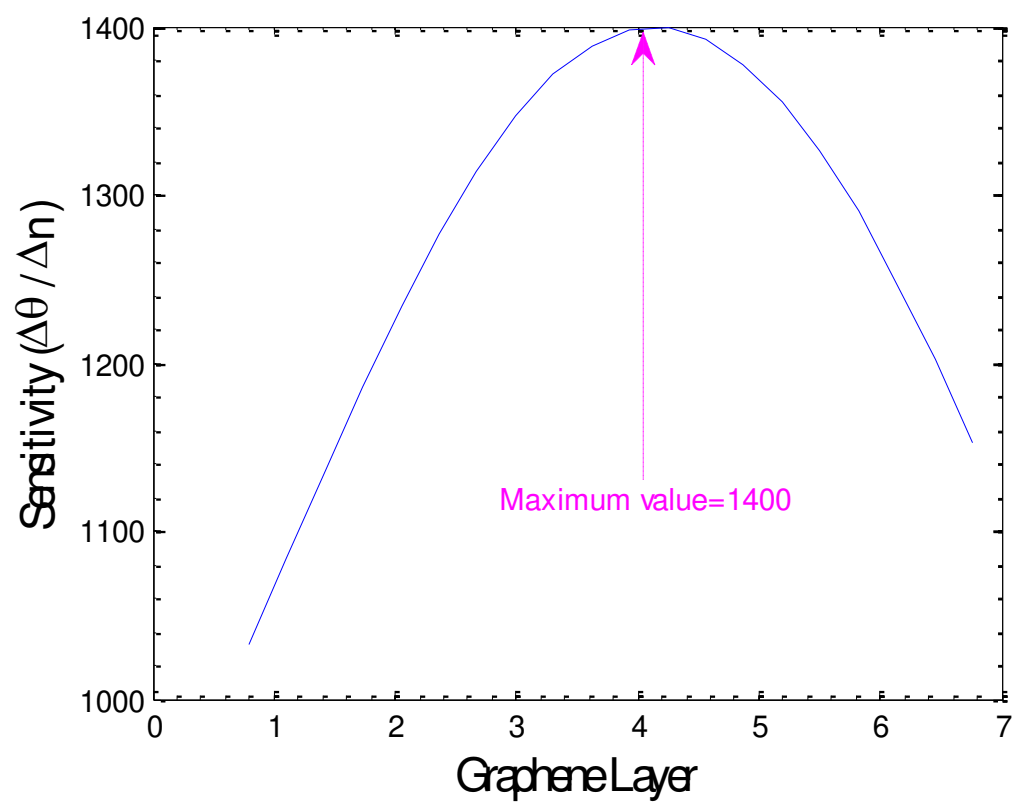

Figure 10. Sensitivity as graphene layers changes.

The experimental part of the proposed structure is contained of AFM results. As can be seen, crystal layers are layered in the proposed structure.

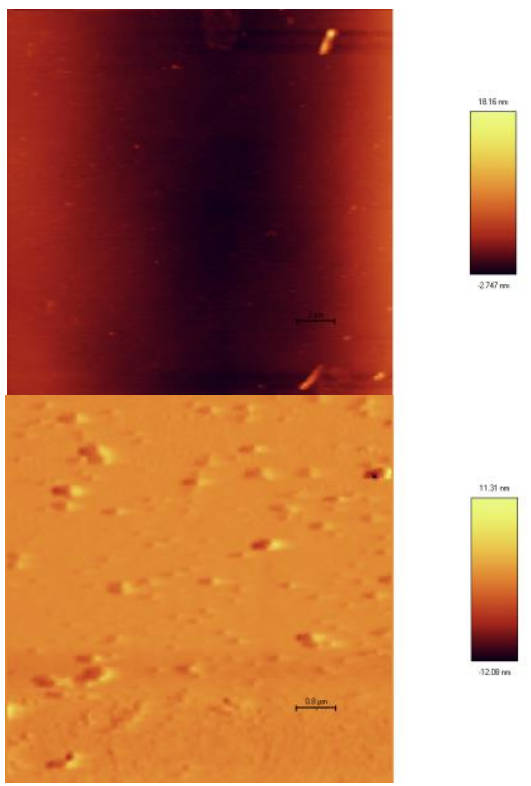

Figure 11. AFM results of the proposed structure.

Finally, we analyze the theoretical and experimental results of the proposed structure. As can be seen, a deviation is observed which related to the roughness of graphene in the fabrication process. 


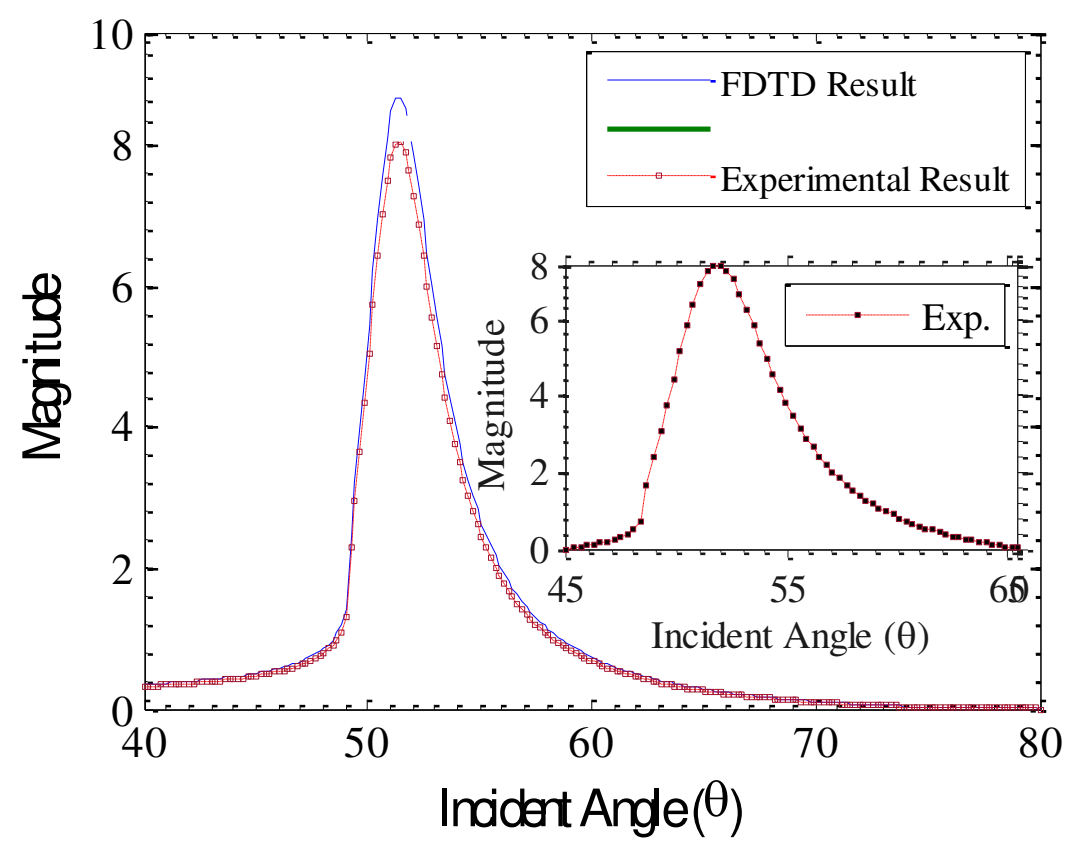

Figure 12. Comparison of theoretical and experimental results of the proposed structure.

\section{Conclusion}

In conclusion, we have proposed a novel PIT sensor, which is composed of a Kretschmann-Raether configuration with graphene/ J-aggregate materials. The proposed device has been investigated numerically and experimentally. The results have shown that the PIT resonance has a sharp contrast ratio compared to previous structures. The maximum sensitivity could reach values as high as $1400 \%$ RIU with a FOM value of about $36 \mathrm{RIU}^{-1}$ near the resonance angle of $53^{\circ}$. Also, the influence of the sensor's structural parameters on performance parameters has been studied in detail. Near the resonance angle, the temperature and magnetic field sensitivity was investigated. Finally, to evaluate the structure, numerical simulation with experimental results are compared. This platform, with strong light-matter interaction, may find many advanced applications, especially in off-chip nanosensors.

\section{Funding}

This research did not receive any specific grant from funding agencies.

\section{Competing interests}

The authors have no conflict of interest to declare.

\section{Availability of data and material}

All data included in this paper are available upon request by contact with the contact corresponding author. 


\section{Code Availability}

All code included in this paper are available upon request by contact with the contact corresponding author.

\section{Author contributions}

Amir Mohammad Rezaei Zangeneh: Software, Data curation. Mohammad Hazhir Mozaffari, and Ali Farmani: Methodology, Investigation. Shahryar Farhadi, Ali Mir, and Ali Farmani: Conceptualization, Methodology, Writing - review and editing. All authors discussed the results and contributed to the final manuscript.

Ethics approval

This is a theoretical work which is used foe sensing platform.

Consent to participate

Not Applicable

Consent for publication

Not Applicable

Acknowledgements (optional)

We would like to thank the reviewers for their thoughtful comments and efforts towards improving our manuscript in advance.

\section{References}

1 Sharafeldin, M., Jones, A. \& Rusling, J. F. 3D-printed biosensor arrays for medical diagnostics. Micromachines 9, 394 (2018).

2 Kaushal, H. \& Kaddoum, G. Applications of lasers for tactical military operations. IEEE Access 5, 20736-20753 (2017).

3 Jogschies, L. et al. Recent developments of magnetoresistive sensors for industrial applications. Sensors 15, 28665-28689 (2015).

4 Verma, N. \& Bhardwaj, A. Biosensor technology for pesticides-a review. Applied biochemistry and biotechnology 175, 3093-3119 (2015).

$5 \quad$ Ferreira, M. F. et al. Roadmap on optical sensors. Journal of Optics 19, 083001 (2017).

$6 \mathrm{Hu}, \mathrm{X}$. et al. Metamaterial absorber integrated microfluidic terahertz sensors. Laser \& Photonics Reviews 10, 962-969 (2016). 
Popa, D. \& Udrea, F. Towards integrated mid-infrared gas sensors. Sensors 19, 2076 (2019).

8 Farmani, A. \& Mir, A. Graphene sensor based on surface plasmon resonance for optical scanning. IEEE Photonics Technology Letters 31, 643-646 (2019).

9 Qian, Y. et al. Intensity-modulated nanoplasmonic interferometric sensor for MMP-9 detection. Lab on a Chip 19, 1267-1276 (2019).

10 Wang, X.-Y. et al. Lasing enhanced surface plasmon resonance sensing. Nanophotonics 6, 472478 (2017).

11 Nazemi, H., Joseph, A., Park, J. \& Emadi, A. Advanced micro-and nano-gas sensor technology: A review. Sensors 19, 1285 (2019).

$12 \mathrm{Li}, \mathrm{B}$. et al. High-sensitivity sensing based on plasmon-induced transparency. IEEE Photonics Journal 7, 1-7 (2015).

13 Wen, L. et al. Multiband and ultrahigh figure-of-merit nanoplasmonic sensing with direct electrical readout in Au-Si nanojunctions. ACS nano 13, 6963-6972 (2019).

14 Shishir, R., Chen, F., Xia, J., Tao, N. \& Ferry, D. Room temperature carrier transport in graphene. Journal of computational electronics 8, 43-50 (2009).

15 Khansili, N., Rattu, G. \& Krishna, P. M. Label-free optical biosensors for food and biological sensor applications. Sensors and Actuators B: Chemical 265, 35-49 (2018).

16 Zhang, R. et al. Label-free electrochemical sensor for cd44 by ligand-protein interaction. Analytical chemistry 91, 7078-7085 (2019).

17 Pirzada, M. \& Altintas, Z. Recent progress in optical sensors for biomedical diagnostics. Micromachines 11, 356 (2020).

18 Del Villar, I., Zubiate, P., Zamarreño, C. R., Arregui, F. J. \& Matias, I. R. Optimization in nanocoated D-shaped optical fiber sensors. Optics express 25, 10743-10756 (2017).

19 TalebiFard, S. et al. Optimized sensitivity of Silicon-on-Insulator (SOI) strip waveguide resonator sensor. Biomedical optics express 8, 500-511 (2017).

20 Lin, Y.-S. et al. Tunable terahertz metamaterial by using three-dimensional double split-ring resonators. Optics \& Laser Technology 112, 215-221 (2019).

21 Lannebère, S., Campione, S., Aradian, A., Albani, M. \& Capolino, F. Artificial magnetism at terahertz frequencies from three-dimensional lattices of tio 2 microspheres accounting for spatial dispersion and magnetoelectric coupling. JOSA B 31, 1078-1086 (2014).

22 Wong, Z. J. et al. Optical and acoustic metamaterials: superlens, negative refractive index and invisibility cloak. Journal of Optics 19, 084007 (2017).

23 Yu-Chieh, C., Ya-Ju, C., Yu-Ching, C., Bo-Zhi, H. \& Chii-Chang, C. A plasmonic refractive index sensor with an ultrabroad dynamic sensing range. Scientific Reports (Nature Publisher Group) 9 (2019).

24 Zhang, X. et al. Refractive index sensor based on fano resonances in plasmonic waveguide with dual side-coupled ring resonators. Photonic Sensors 8, 367-374 (2018).

25 Willets, K. A. \& Van Duyne, R. P. Localized surface plasmon resonance spectroscopy and sensing. Annu. Rev. Phys. Chem. 58, 267-297 (2007).

26 Zhong, Y., Malagari, S. D., Hamilton, T. \& Wasserman, D. M. Review of mid-infrared plasmonic materials. Journal of Nanophotonics 9, 093791 (2015).

27 Liu, Z., Liu, G., Liu, X. \& Fu, G. Plasmonic sensors with an ultra-high figure of merit. Nanotechnology 31, 115208 (2019).

28 Chen, J. et al. Engineering the magnetic plasmon resonances of metamaterials for high-quality sensing. Optics express 25, 3675-3681 (2017).

29 Salemizadeh, M., Mahani, F. F. \& Mokhtari, A. Design of aluminum-based nanoring arrays for realizing efficient plasmonic sensors. JOSA B 36, 786-793 (2019). 
Yang, L. et al. Characteristics of multiple Fano resonances in waveguide-coupled surface plasmon resonance sensors based on waveguide theory. Scientific reports 8, 1-10 (2018).

31 Lu, H., Gan, X., Mao, D., Jia, B. \& Zhao, J. Flexibly tunable high-quality-factor induced transparency in plasmonic systems. Scientific reports 8, 1-9 (2018).

32 Liu, L. et al. Multiple detuned-resonator induced transparencies in MIM plasmonic waveguide. Optics Communications 418, 27-31 (2018).

33 Liu, B. et al. A plasmonic sensor array with ultrahigh figures of merit and resonance linewidths down to $3 \mathrm{~nm}$. Advanced Materials 30, 1706031 (2018).

$34 \mathrm{He}, \mathrm{Z}$. et al. Tunable multi-switching in plasmonic waveguide with Kerr nonlinear resonator. Scientific reports 5, 1-9 (2015).

$35 \mathrm{Xu}, \mathrm{H}$. et al. Influential and theoretical analysis of nano-defect in the stub resonator. Scientific reports 6, 1-7 (2016).

36 Wang, G., Zhang, W., Gong, Y. \& Liang, J. Tunable slow light based on plasmon-induced transparency in dual-stub-coupled waveguide. IEEE Photonics Technology Letters 27, 89-92 (2014).

37 Hokmabadi, M. P., Philip, E., Rivera, E., Kung, P. \& Kim, S. M. Plasmon-induced transparency by hybridizing concentric-twisted double split ring resonators. Scientific reports 5, 1-11 (2015). Matsunaga, K., Hirai, Y., Neo, Y., Matsumoto, T. \& Tomita, M. Tailored plasmon-induced transparency in attenuated total reflection response in a metal-insulator-metal structure. Scientific reports 7, 1-9 (2017).

39 Chen, Z., Cui, L., Song, X., Yu, L. \& Xiao, J. High sensitivity plasmonic sensing based on Fano interference in a rectangular ring waveguide. Optics Communications 340, 1-4 (2015). Chen, J. et al. Dielectric waveguide-enhanced localized surface plasmon resonance refractive index sensing. Optical Materials Express 8, 342-347 (2018).

41 Yin, X. \& Hesselink, L. Goos-Hänchen shift surface plasmon resonance sensor. Applied physics letters 89, 261108 (2006).

42 Löffler, W. et al. Polarization-dependent Goos-Hänchen shift at a graded dielectric interface. Optics communications 283, 3367-3370 (2010).

43 Qamar, S. \& Zubairy, M. S. Coherent control of the Goos-Hänchen shift. Physical Review A 81, 023821 (2010).

44 Olaya, C. M., Hayazawa, N., Hermosa, N. \& Tanaka, T. Angular Goos-Hänchen Shift Sensor Using a Gold Film Enhanced by Surface Plasmon Resonance. The Journal of Physical Chemistry $A$ (2020).

45 Bai, Z., Huang, G., Liu, L. \& Zhang, S. Giant Kerr nonlinearity and low-power gigahertz solitons via plasmon-induced transparency. Scientific reports 5, 1-11 (2015).

46 Butt, M., Khonina, S. \& Kazanskiy, N. Hybrid plasmonic waveguide-assisted Metal-InsulatorMetal ring resonator for refractive index sensing. Journal of Modern Optics 65, 1135-1140 (2018).

47 Tassin, P., Koschny, T., Kafesaki, M. \& Soukoulis, C. M. A comparison of graphene, superconductors and metals as conductors for metamaterials and plasmonics. Nature Photonics 6, 259 (2012).

48 Zhang, Y. et al. Direct observation of a widely tunable bandgap in bilayer graphene. Nature 459, 820-823 (2009).

49 Novoselov, K. S. et al. A roadmap for graphene. nature 490, 192-200 (2012).

50 Falkovsky, L. in Journal of Physics: conference series. 012004 (IOP Publishing).

$51 \mathrm{He}, \mathrm{Y}$. et al. Graphene plasmonically induced analogue of tunable electromagnetically induced transparency without structurally or spatially asymmetry. Scientific reports 9, 1-9 (2019). 
52 Ma, Q., Dai, J., Luo, A. \& Hong, W. Numerical and theoretical study of Tunable Plasmonically induced transparency effect based on bright-dark mode coupling in Graphene Metasurface. Nanomaterials 10, 232 (2020). 